\title{
Shift of Keratin Expression Profile in End-stage Kidney Increases the Risk of Tumor Development
}

\author{
DONAT PETER SARLOS ${ }^{1}$, LEHEL PETERFI $^{1}$, ARPAD SZANTO $^{1}$ and GYULA KOVACS ${ }^{1,2}$ \\ ${ }^{1}$ Department of Urology, Medical School, University of Pecs, Pecs, Hungary; \\ ${ }^{2}$ Medical Faculty, Ruprecht-Karls-University, Heidelberg, Germany
}

\begin{abstract}
Background/Aim: Pre-neoplastic lesions and renal cell tumors of distinct pheno- and genotypes occur frequently in end-stage kidneys. The aim of this study was to investigate the role of KRT7 and KRT19 in this process, the expression of which was previously detected by Affymetrix GeneChip analysis. Patients and Methods: Twelve end-stage kidneys were analyzed to find pre-neoplastic lesions and tumors and expression of KRT7 and KRT19 was examined by immunohistochemistry. Results: A total of 17 tumors, 149 preneoplastic lesions, 179 simple or proliferative cysts $>2 \mathrm{~mm}$ were identified. Diffuse expression of KRT7 and KRT19 was seen in all end-stage kidneys as well as in the vast majority of cysts, pre-neoplastic lesions and tumors. Conclusion: Our data indicates that de novo expression of KRT7 and KRT19 resulting in altered plasticity and stem cell characteristics of epithelial cells might be a crucial factor in increasing the risk of tumor development in end-stage kidneys.
\end{abstract}

Chronic renal disease (CRD) is characterized by a decrease in kidney function to a degree requiring renal replacement therapy (RRT). The long survival of patients with nonfunctioning kidneys results in remodeling of kidney structure, so-called end-stage renal disease (ESRD). In spite of atrophic structures, ESRD kidney shows a remarkable proliferative activity (1). In sclerotic end-stage kidneys diffuse cystic changes may develop, especially after long intermittent maintenance haemodialysis, and this condition is defined as acquired cystic renal disease (ACRD) (2). Remodelling of kidneys in ESRD/ACRD is frequently accompanied by preneoplastic lesions and renal cell tumors (RCT) of distinct pheno- and genotype (3-8). Clinically recognized renal cell

Correspondence to: Gyula Kovacs, Department of Urology, Medical School, University of Pecs, Munkacsy M. u. 2, H-7621 Pecs, Hungary. Tel: +36 72507334, Fax: +36 72242374, e-mail: G.Kovacs@gmx.de, gyula.kovacs@urz.uni-heidelberg.de

Key Words: End-stage renal disease, KRT7, KRT19, immunohistochemistry, tumor development. carcinoma (RCC) has been found in $3.8 \%$ of native end-stage kidneys which contrasts with a detection rate of $0.04 \%$ to $0.3 \%$ lifetime risk of developing RCC in the general population $(9,10)$. Malignancy is currently reported as a cause of death in 3 to $4 \%$ of ESRD/ACRD patients, largely as the result of an increasing incidence of RCC (11).

The underlying molecular mechanism of structural remodeling of ESRD/ACRD kidneys, as well as of development of tumors is not yet known. RCTs arising in the general population are characterized by specific genomic alterations. It has been proposed that in ESDR/ACRD the microenvironment may trigger the development of tumors of unusual pheno- and genotype (12). Expression of growth factors and hypoxemia have been suggested to be responsible for cystic changes and tumors (13-15). Global gene expression analysis disclosed a Bunique gene expression signature including a group of cytokines indicating an inflammatory microenvironment in ESRD/ACRD kidneys (14). Another well-defined group of functionally related genes expressed in ESRD/ACRD kidneys encodes intermediate filament proteins including the keratins KRT7 and KRT19 (14). It was demonstrated that KRT7 and KRT19 are up-regulated during renal epithelial injury (16). KRT19 has also been described as a putative marker of epidermal stem cells and its overexpression was associated with metastatic capacity of tumours (17-19).

The aim of this study was to analyse the morphological changes and KRT7 and KRT19 expression in ESRD/ACRD kidneys, pre-neoplastic lesions and associated tumours by immunohistochemistry. A diffuse KRT7 and KRT19 expression was found in ESRD/ACRD kidneys and associated tumors indicating their role in structural remodelling and tumorigenesis.

\section{Materials and Methods}

Tissue samples. From 11 ESRD/ACRD cases 12 entire kidneys were available and processed in several hundreds of paraffin blocks for histological analysis. ACRD was diagnosed when the secondary cystic changes replaced at least $40 \%$ of the kidney parenchyma. The 
hematoxylin and eosin $(\mathrm{H} \& \mathrm{E})$ stained slides were scored for cysts, small precursor lesions and tumors. The diagnosis of tumors was established according to the Heidelberg Classification (20) and Tickoo et al. (5). Tissue multi array (TMA) was constructed from paraffin embedded ESRD/ACRD associated tumors after marking the areas of interest on H\&E-stained slides by one of the authors (GK). Core biopsies of $0.6 \mathrm{~mm}$ in diameter were placed within a recipient block by Manual Tissue Arrayer (MTA1, Beecher Instruments, Inc., Sun Prairie, WI, USA). The collection and use of tissue samples for this study was approved by the Ethics Committees of the University of Heidelberg, Germany and University of Pecs, Hungary.

Immunohistochemistry. Paraffin blocks of normal and ESRD/ARCD kidneys as well as a TMA containing ESRD/ARCD associated tumours were used for immunohistochemistry. Serial sections of ESRD/ACRD kidneys were used to compare the results obtained by different antibodies. After dewaxing and rehydration of the slides, antigen de-masking was performed in $10 \mathrm{mM}$ sodium citrate buffer, pH 6,0 or TE buffer, $\mathrm{pH} 9,0$ in 2100-Retriever (Pick-Cell Laboratories, Amsterdam, The Netherlands). Endogenous peroxidase activity and unspecific binding sites were blocked with $0.3 \%$ hydrogen peroxide containing $1 \%$ normal horse serum for 10 min at room temperature. Slides were incubated overnight with KRT7 (mouse monoclonal antibody, ab9021, Abcam, Cambridge, UK) in 1:300 dilution, KRT19 (rabbit polyclonal antibody, ab14363-1, Abcam,) in 1:100 dilution, TMEM27 (rabbit polyclonal antibody, ab200664, Abcam,) in 1:1,000 dilution and AQP2 (rabbit polyclonal antibody, PA5-38004, Thermo Fisher, Budapest, Hungary) in 1:200 dilution. HRP conjugated secondary antibody (MACH4 Universal HRP-Polymer, Biocare Medical, Concord, CA, USA) was applied for $30 \mathrm{~min}$. The signal was visualized with AEC (Amino-ethylcarbazol) and DAB (3,3'-Diaminobenzidin). Tissue sections were counterstained with Mayer's haematoxylin. In negative control the primary antibody was omitted.

\section{Results}

Cysts, precursor lesions and tumours in ESRD/ACRD. Analysis of 393 paraffin blocks obtained from 12 entire kidneys of 11 patients with ESRD/ACRD revealed 17 tumors with a size of $2-41 \mathrm{~mm}$ in diameter (Table I). The size of main tumours varied between 8 and $41 \mathrm{~mm}$. Five tumours were diagnosed as papillary renal cell tumor (pRCT) and another 6 as conventional renal cell carcinoma (cRCC), all showing similar histology to those occurring in the general population. One oncocytoma, two ACRD-associated eosinophil-vacuolated tumors, two chromophobe-like tumors, and one clear cell papillary RCC were also found (Table I).

By detailed microscopic analysis of the 393 slides we detected 143 small cysts, 26 proliferative cysts with solidpapillary growth of cells within the cysts. Ten of the proliferative cysts displayed solid growth of large eosinophil cells with cytoplasmic vacuoles. Overall, 65 small papillary precursor lesions of small or medium sized cells revealed similar growth pattern, which was earlier described in kidneys with papillary RCC in the general population (21). The overwhelming majority of the 42 pre-neoplastic lesions with chromophobe RCC-like pattern occurred in the two kidneys of one patient (173R and 173L). Nearly all of the 24 small solid lesions with large eosinophil vacuolated cells were located in one kidney (209). The number of cysts and distinct types of pre-neoplastic lesions are shown in Table II. A good correlation was found between the phenotype of preneoplastic lesions and tumors, the majority of pre-neoplastic lesions displayed a similar morphology to the tumor observed in the same kidney (Figure 1).

Expression of KRT7 and KRT 19 in ESRD/ACRD kidneys, pre-neoplastic lesions and tumours. In normal kidney KRT7 and KRT19 proteins were expressed in principal cells of the collecting duct. However, in ESRD/ACRD kidneys a diffuse $K R T 7$ and KRT19 staining was seen in small atrophic as well as in dilated tubules lined with flat or cuboidal epithelial cells (Figure 2A and B). To clear the origin of cells stained positively with both KRT7 and KRT19, we have analysed ESRD/ACRD kidneys for expression of structural proteins of proximal tubules (TMEM27) and principal cells of the collecting duct (AQP2). No expression of TMEM27 and AQP2 or expression only in single tubules were observed (Figure 2C and D).

There was a strong immunostaining in dilated tubules showing papillary growth within the lumen (Figure 2E). All papillary RCTs developed in ESRD/ACRD kidneys showed diffuse positivity with KRT7 antibody (Figure 2F). A membrane attenuated positive staining was seen in each small chromophobe-like pre-neoplastic lesion in kidneys $173 \mathrm{R}$ and $173 \mathrm{~L}$ as well as in the chromophobe-like cancer (Figure 2G and H). ACRD associated eosinophil vacuolated tumors displayed only scattered positivity with KRT7. Conventional and clear cell papillary RCC were consequently negative for KRT7.

All cells of tubular-papillary growing tumours including those with broad papillary stalk displaying strong inflammation exhibited a strong KRT19 positive immunostaining (Figure 2I and J). Proliferative cystic pre-neoplastic lesions for ACRDassociated eosinophilic-vacuolated tumor and also the tumor itself displayed focal cytoplasmic staining for KRT19 (Figure $2 \mathrm{~K})$. The chromophobe-like precursor lesions and tumours also showed a strong KRT19 immunoreaction. Of interest, 4 of the 6 conventional RCCs showed scattered positivity for KRT19 (Figure 2L), whereas the clear cell papillary RCC was negative.

\section{Discussion}

In chronic renal disease nephrons gradually lose their function and undergo an extreme structural remodeling. There is a long-debated question on the origin of atrophic 
Table I. Pertinent clinical and histological data of ESRD/ACRD tumours.

\begin{tabular}{|c|c|c|c|c|c|}
\hline Tumour samples & Patient age/gender & Renal disease & Size of kidney $(\mathrm{cm})$ & Size of tumor $(\mathrm{cm})$ & Histological diagnosis \\
\hline $94 \mathrm{~A}$ & $76 / \mathrm{M}$ & ESRD/NS & $9.5 \times 6 \times 3$ & 0.8 & $\mathrm{cRCC}$ \\
\hline $94 \mathrm{~B}$ & & & & 0.2 & $\mathrm{pRCT}$ \\
\hline $94 \mathrm{C}$ & & & & 0.3 & pRCT \\
\hline 105 & $71 / \mathrm{F}$ & $\mathrm{ACRD} / ?$ & $12 \times 6.5 \times 5$ & 4.0 & RO \\
\hline $123 \mathrm{~A}$ & $77 / \mathrm{M}$ & $\mathrm{ACRD} / \mathrm{GN}$ & $15 \times 8 \times 7$ & 3.1 & ACRD-T \\
\hline $123 \mathrm{~B}$ & & & & 2.6 & $\mathrm{cRCC}$ \\
\hline $123 \mathrm{C}$ & & & & 2.2 & ccpRCC \\
\hline 137 & $61 / \mathrm{M}$ & ESRD/NS & $6.5 \times 3.0 \times 3.0$ & 3.0 & cRCC \\
\hline $173 \mathrm{~L}$ & $68 / \mathrm{M}$ & $\mathrm{ESRD} / \mathrm{GN}$ & $5.5 \times 3.0 \times 1.5$ & 2.5 & chRCC-like \\
\hline $173 \mathrm{R}$ & & & $5.5 \times 3.0 \times 2.0$ & 1.5 & chRCC-like \\
\hline 192 & 49/M & ACRD/NS & $10 \times 5.5 \times 3$ & 3.5 & cRCC \\
\hline 195 & $43 / \mathrm{M}$ & $\mathrm{ACRD} / ?$ & $9 \times 5 \times 4$ & 3.0 & $\mathrm{cRCC}$ \\
\hline $203 \mathrm{~A}$ & $54 / \mathrm{M}$ & $\mathrm{ESRD} / ?$ & $7 \times 3.5 \times 2.5$ & 4.1 & pRCT \\
\hline $203 \mathrm{E}$ & & & & 0.3 & pRCT \\
\hline 209 & $49 / \mathrm{M}$ & $\mathrm{ACRD} / \mathrm{GN}$ & $9.5 \times 5.5 \times 4$ & 3.2 & ACRD-T \\
\hline 217 & $71 / \mathrm{M}$ & ESRD/? & $6.5 \times 3.5 \times 3$ & 2.8 & $\mathrm{cRCC}$ \\
\hline 1926 & $33 / \mathrm{M}$ & ACRD/? & $10 \times 5 \times 4$ & 3.5 & pRCT \\
\hline
\end{tabular}

NS: Nephrosclerosis; ?: unknown; GN: glomerulonephritis; cRCC: conventional RCC; pRCT: papillary RCT; RO: renal oncocytoma; ACRD-T: ACRD associated large eosinophilic cells with cytoplasmic vacuoles; ccpRCC: clear cell papillary RCC; chRCC-like: similar morphology to chRCC.

Table II. Type and number of precuros lesions in ESRD/ACRD.

\begin{tabular}{lcrrrrrr}
\hline Kidney samples & Nr. of blocks & Cyst & p-cyst & Conv & Pap & ch-like & 1-eos \\
\hline 94 & 27 & 11 & 3 & 0 & $\mathbf{2 1}$ & 2 & 1 \\
105 & 21 & 13 & 2 & 0 & 5 & 0 & 0 \\
123 & 95 & 18 & $4 *$ & 0 & $\mathbf{1 3}$ & 0 & 0 \\
137 & 32 & 3 & 0 & 3 & 2 & 0 & $\mathbf{4}$ \\
$173 \mathrm{R}$ & 28 & 14 & 2 & 0 & 2 & $\mathbf{1 9}$ & 0 \\
$173 \mathrm{~L}$ & 24 & 7 & 0 & 0 & 0 & 0 \\
192 & 23 & 6 & 0 & $\mathbf{4}$ & 0 & 0 \\
195 & 29 & 38 & 12 & $\mathbf{5}$ & 2 & 0 & 0 \\
203 & 25 & 11 & 2 & 1 & 5 & 0 & 0 \\
209 & 40 & 12 & $6 *$ & 0 & 1 & 0 & 0 \\
217 & 27 & 2 & 3 & $\mathbf{4}$ & 1 & 0 & 0 \\
1926 & 22 & 8 & 2 & 0 & $\mathbf{1 2}$ & 0 & 0 \\
\hline
\end{tabular}

p-cyst: Proliferative cyst; conv: conventional-like lesion; pap: papillary lesion; ch-like: chromophobe-like lesion; 1-eos: large eosinophilic cells with vacuoles; ccpap: clear cell papillary lesion; *proliferative cyst with large vacuolated cells. The dominant type of precursor lesion is marked in bold.

and proliferating cells in ESRD/ACRD kidneys $(1,14)$. KRT7 and KRT19 are expressed only in principal cells of the collecting duct and ascending loop of Henle. We found a diffuse positivity for KRT7 and KRT19 in atrophic tubules and single epithelial cells embedded in fibrous stroma as well as in dilated and cystic tubules. In this study, TMEM27 and AQP2 antibodies, which recognize structural proteins of proximal tubules and principal cells of the collecting duct, respectively, stained only single tubules in ESRD/ACRD kidneys. It was recently documented that SCEL, a marker of distal tubular cells, shows diffuse immune-reaction in nearly all cells of ESRD/ACRD kidneys (22). Therefore, we suggest that the vast majority of solid epithelial cell clusters, aberrant tubular and cystic structures in ESRD/ACRD kidneys correspond to proliferating distal tubules.

Strong arterial and arteriolar sclerosis leads to slow or blocked blood flow in ESRD kidneys (23). The reduced oxygen and nutrient delivery results in damage of epithelial cells especially of proximal tubules, which cannot easily convert from oxidative to glycolytic metabolism. Thus, 

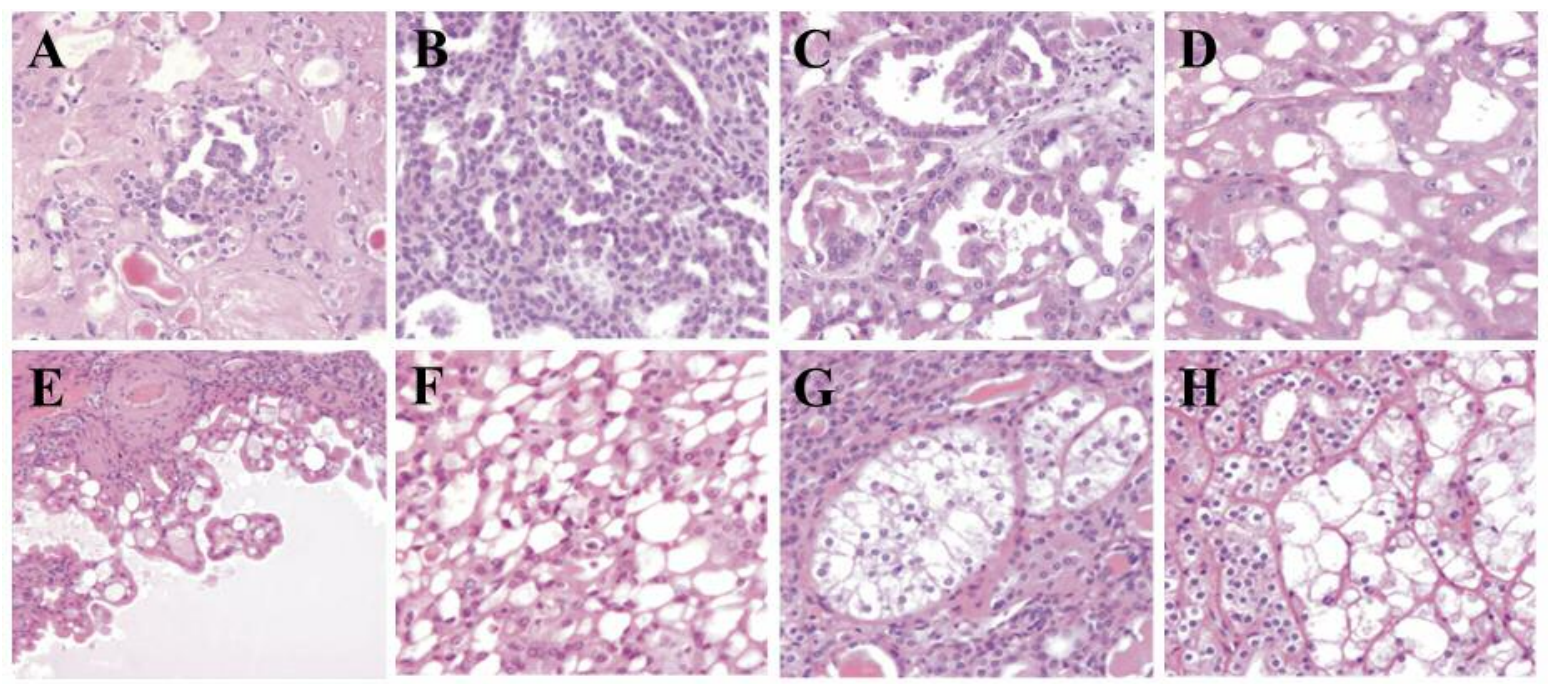

Figure 1. Morphological heterogeneity of precursor lesions and ESRD/ACRD tumors. A, Tubulopapillary growth of small-cell pre-neoplastic lesion (A) and papillary $R C T(B)$. A pre-neoplastic lesion showing a transition from small-cell papillary morphology towards large eosinophilic cells $(C)$ and the ACRD-associated vacuolated eosinophil tumor $(D)$ of the same kidney. A proliferative cyst lined with large vacuolated eosinophil cells $(E)$ and corresponding tumor $(F)$. Small chromophobe-like pre-neoplastic lesion $(G)$ and tumor $(H)$.
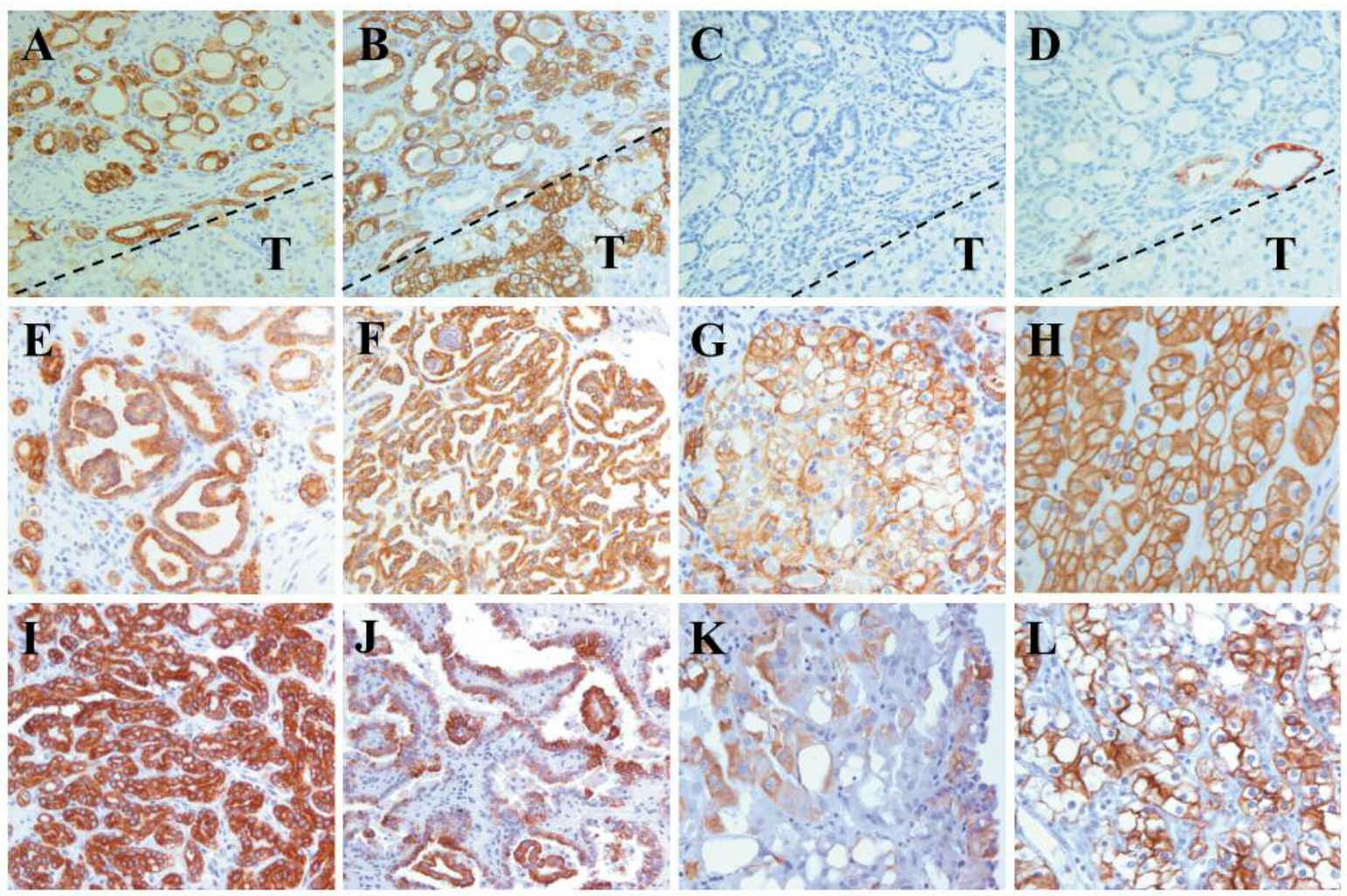

Figure 2. Expression of KRT7 and KRT19 in ESRD kidney, pre-neoplastic lesions and tumors. Diffuse KRT7 (A) and KRT19 (B) positivity but no or scattered staining with AQP2 $(C)$ and TMEM27 (D); the corresponding tumor $(T)$ shows positive reaction with KRT19. KRT7 positive immunoreaction in a papillary preneoplastic lesion $(E)$, papillary $R C T(F)$ as well as in a small chromophobe-like lesion $(G)$ and corresponding tumor $(H)$. Note the strong KRT19 staining in a tubulopapillary $(I)$ and papillary $(J) R C T$, scattered positivity in a ACRD-associated tumor (K) and also in a conventional $R C C(L)$. 
proximal tubular cells are not only the victims but actively participate in the inflammatory response to ischemia by releasing cytokines and chemokines which play an important role in progressive fibrosis which in turn increases the hypoxemic stress $(24,25)$. Cells of the distal tubules are more resistant to hypoxia and have greater capacity to shift from oxidative to glycolytic metabolism, which may explain the survival and preferential proliferation of cells of distal tubules.

Corresponding to the hypoxemic stage, high expression of hypoxia inducible gene HIG2, HIF1A and also NFkB triggering the expression of pro-inflammatory and inflammatory proteins, has been documented in ESRD/ACRD kidneys, cysts and epithelial hyperplasia (25). HIF1A is also expressed in conventional RCC whereas HIG2 in papillary RCTs. Using global gene expression analysis two groups of functionally related genes have been detected in ESRD/ACRD kidneys (14). The high expression of cytokines including IL-6, IL-8 and their receptors indicated a strong inflammatory microenvironment (14). The other group of functionally related genes included KRT7 and KRT19, which are known to be associated with increased plasticity of epithelial cells. Recently, it was also shown that expression of IL-6, TNFa and NFkB leads to upregulation of cytokeratins in experimental renal disease models (26).

Highly specialized epithelial cells of kidney express, at normal state, only KRT8 and KRT18, which are crucial in keeping the integrity and mechanical stability of tubular epithelial cells. Recent studies showed that this structural support may be modulated to accommodate the changing needs of cells and pointed out a novel role of intermediate filaments influencing cell growth and death through dynamic interactions with non-structural proteins. Keratins are not only structural cell markers but are also stress-responsive proteins in tubular cells (26). For example, KRT7 and KRT19 are upregulated during renal epithelial injury and repair (16). Thus, upon various types of stress such as hypoxemia, inflammation, renal tubular injury or atrophy, each of them occurring in ESRD/ACRD, epithelial cells may switch on expression of KRT7 and KRT19, which appears to correlate with reduction in the degree of terminal differentiation.

A diffuse expression of KRT7 and KRT19 was found not only in ESRD/ACRD kidneys, cysts, pre-neoplastic lesions but also in tumors of distinct histological types. KRT19 has been described as a putative marker of epidermal stem cells (17). The increased expression of KRT19 shows a good correlation with the invasive growth and metastatic potential of liver and cancer $(18,19)$. Serum levels of CYFRA 21-1, a KRT19 fragment, can be used as a tumour marker in variety of cancers such as non-small cell lung cancer, breast, ovarian, liver and bladder cancer to detect tumour cell dissemination and presence of metastasis $(27,28)$.
In summary, our results indicate that tubular cells of endstage kidneys suspend their program of terminal differentiation and shift their keratin expression profile from KRT8 and KRT18 to KRT7 and KRT19 which allows them to alter their shape and to increase their plasticity necessary for the remodeling and tumorigenic processes. ESRD/ACRD is associated with large number of proliferative cysts and pre-neoplastic lesions with distinct cellular characteristics similar to those observed in tumors from the corresponding kidney. Previous and present data indicates that ESRD/ACRD is a novel disease. The hypoxemic and inflammatory microenvironment alters the plasticity and leads to stem cell characteristics of epithelial cells which might be crucial factor in tumor development.

\section{Conflicts of Interest}

Authors have no conflicts of interest to declare.

\section{Acknowledgements}

This work was supported by a grant of PTE-AOK KA-2016-01. The Authors thank Drs. G. Staehler (Department of Urology, University of Heidelberg, Germany), B. Schulze-Brüggemann (Department of Urology, District Hospital Bad-Hersfeld, Germany), D. Ferluga (Insitute of Pathology, University of Ljubljana, Slovenia), and Mr. D. Cranston (Department of Urology, Radcliffe Hospital, Cambridge, UK) for making clinical material available from end-stage kidneys.

\section{References}

1 Nadasdy T, Laszik Z, Lajoie G, Blick KE, Wheeler DE and Silva FG: Proliferative activity of cyst epithelium in human renal cystic diseases. J Am Soc Nephrol 5: 1462-1468, 1995.

2 Dunnill MS, Millard PR and Oliver D: Acquired cystic disease of the kidneys: A hazard of long-term intermittent maintenance haemodialysis. J Clin Pathol 30: 868-877, 1997.

3 Ishikawa I and Kovacs G: High incidence of papillary renal cell tumors in patients on chronic haemodialysis. Histopathology 22: 135-139, 1993.

4 Chudek J, Herbers J, Wilhelm M, Kenck C, Bugert P, Ritz E, Waldman $\mathrm{F}$ and Kovacs G: The genetics and morphology of renal cell tumors in end-stage renal failure may differ from those occurring in the general population. J Am Soc Nephrol 9: 1045$1051,1998$.

5 Tickoo SK, dePeralta-Venturina MN, Harik LR, Worcester HD, Salama ME, Young AN, Moch H and Amin MB: Spectrum of epithelial neoplasms in end-stage renal disease: an experience from 66 tumor-bearing kidneys with emphasis on histologic patterns distinct from those in sporadic adult renal neoplasia. Am J Surg Pathol 30: 141-153, 2006.

6 Pan CC, Chen YJ, Chang LC, Chang YH and Ho DM: Immunohistochemical and molecular genetic profiling of acquired cystic disease-associated renal cell carcinoma. Histopathology 55: 145-153, 2009.

7 Kuntz E, Yusenko MV, Nagy A and Kovacs G: Oligoarray-CGH of renal cell tumors developed in patients with acquired cystic renal disease. Hum Pathol 41: 1345-1349, 2011. 
8 Inoue T, Matsuura K, Yoshimoto T, Nguyen LT, Tsukamoto Y, Nakada C, Hijiya N, Narimatsu T, Nomura T, Sato F, Nagashima Y, Kashima K, Hatakeyama S, Ohyama C, Numakura K, Habuchi T, Nakagawa M, Seto M, Mimata $\mathrm{H}$ and Moriyama M: Genomic profiling of renal cell carcinoma in patients with endstage renal disease. Cancer Sci 103: 569-576, 2012.

9 Chow WH, Dong LM and Devesa SS: Epidemiology and risk factors for kidney cancer. Nat Rev Urol 7: 245-257, 2010.

10 Butler AM, Olshan AF, Kshirsagar AV, Edwards JK, Nielsen ME, Wheeler SB and Brookhart MA: Cancer incidence among US Medicare ESRD patients receiving haemodialysis, 19962009. Am J Kidney Dis 65: 763-672, 2015.

11 Denton MD, Magee CC, Ovuworie C, Mauiyyedi S, Pascual M, Colvin RB, Cosimi AB, Tolkoff-Rubin N: Prevalence of renal cell carcinoma in patients with ESRD pre-transplantation: A pathologic analysis. Kidney Int 61: 2201-2209, 2002.

12 Kovacs G: High frequency of papillary renal cell tumours in end-stage kidneys - is there a molecular genetic explanation? Nephrol Dial Transplant 10: 593-596, 1995.

13 Konda R, Sato H, Hatafuku F, Nozawa T, Ioritani N and Fujioka $\mathrm{T}$ : Expression of hepatocyte growth factor and its receptor c-met in acquired renal cystic disease associated with renal cell carcinoma. J Urol 171: 2166-2170, 2004.

14 Nagy A, Walter E, Zubakov D and Kovacs G: High risk of development of renal cell tumor in end-stage kidney disease: the role of microenvironment. Tumor Biol 37: 9511-9519, 2016.

15 Cohen EP and Elliott WC: The role of ischemia in acquired cystic kidney disease. Am J Kidney Dis 15: 55-60, 1990.

16 Djudjaj S, Papasotiriou M, Büpow RD, Wagnerova A, Lindenmeyer MT, Cohen CD, Strnad P, Goumenos DS, Floege $\mathrm{J}$ and Boor P: Keratins are novel markers of renal epithelial injury. Kidney International 89: 792-808, 2016.

17 Commo S, Gaillard O and Bernard BA: The human hair follicle contains two distinct K19 positive compartments in the outer root sheath: a unifying hypothesis for stem cell reservoir? Differentiation 66: 157-164, 2000.

18 Kabir NN, Rönnstrand L and Kazi JU: Keratin 19 expression correlates with poor prognosis in breast cancer. Mol Biol Rep 41: 7729-7735, 2014.

19 Govaere O, Komuta M, Berkers J, Spee B, Janssen C, de Luca F, Katoonizadeh A, Wouters J, van Kempen LC, Durnez A, Verslype C, De Kock J, Rogiers V, van Grunsven LA, Topal B, Pirenne J, Vankelecom H, Nevens F, van den Oord J, Pinzani M and Roskams T: Keratin 19: a key role player in the invasion of human hepatocellular carcinomas. Gut 63: 674-685, 2014.
20 Kovacs G, Akhtar M, Beckwith BJ, Bugert P, Cooper CS, Delahunt B, Eble JN, Fleming S, Ljungberg B, Medeiros LJ, Moch H, Reuter VE, Ritz E, Roos G, Schmidt D, Srigley JR, Störkel S, van den Berg E and Zbar B: The Heidelberg classification of renal cell tumours. J Pathol 183: 131-133, 1997.

21 Banyai D, Banyai D, Sarlos DP, Anetta Nagy A and Kovacs G: Recalling Cohnheim's theory: Papillary renal cell tumor as a model of tumorigenesis from impaired embryonal development to malignant tumors in adults. Int J Biol Sci 14: 784-790, 2018.

22 Nagy A, Banyai D, Semjen D, Beothe T and Kovacs G: Sciellin is a marker for papillary renal cell tumours. Virchows Arch 467: 695-700, 2015

23 Hughson MD, Hennigar GR and McManus JFA: Atypical cysts, acquired renal cystic disease, and renal cell tumors in end-stage dialysis kidneys. Lab Invest 42: 475-481, 1980.

24 Daha MR and van Kooten C: Is the proximal tubular cell a proinflammatory cell? Nephrol Dial Transplant (6): 41-43, 2000.

25 Konda R, Sugimura J, Sohma F, Katagiri T, Nakamura Y and Fujioka T: Over expression of hypoxia-inducible protein 2, hypoxia-inducible factor-1a and nuclear factor $\mathrm{kB}$ is putatively involved in acquired renal cyst formation and subsequent tumor transformation in patients with end-stage renal failure. J Urol 180: 481-485, 2008

26 Toivola DM, Strnad P, Habtezion A and Omary MB: Intermediate filaments take the heat as stress proteins. Trends Cell Biol 20: 79-91, 2009.

27 Huang YL, Chen J, Yan W, Zang D, Qin Q, Deng AM: Diagnostic accuracy of cytokeratin-19 fragment (CYFRA 21-1) for bladder cancer: a systematic review and metaanalysis. Tumor Biol 36: 3137-3145, 2015.

28 Kolostova K, Rzechonek A, Schützner J, Grill R, Lischke R, Hladik P, Simonek J and Bobek V: Circulating tumor cells as an auxiliary diagnostic tool in surgery. In Vivo 31: 1197-1202, 2017. 